\title{
Salivary and Serum Antioxidant Activity in Patients with Acute Coronary Syndrome
}

\author{
Gulati Ya, Narain VS ${ }^{\mathrm{b}}$, Saxena $\mathrm{AK}^{\mathrm{a}}$, Nigam PK ${ }^{\mathrm{b}}$, Ali B ${ }^{\mathrm{a}}$ \\ a Department Of Pharmacology \& Therapeutics, C.S.M. Medical University (Upgraded King George's \\ Medical College), Lucknow 226003 (U.P.), India \\ ${ }^{b}$ Department Of Cardiology, C.S.M. Medical University, (Upgraded King George's Medical College), \\ Lucknow 226003 (U.P.), India
}

\begin{abstract}
Introduction: Oxidative stress has been implicated in the pathogenesis of many diseases, including cardiovascular diseases. However, much less is understood about the redox status of patients with acute coronary syndrome (ACS). The present study was, therefore, designed to evaluate the redox status of patients with ACS prior to and following therapy as compared to age-matched healthy individuals by monitoring the antioxidant potential of serum and saliva as biomarkers of oxidative stress and evaluating their likely correlation with the clinical status of the patients. Methods: A total of 33 patients (29-78 year old, male) with the diagnosis of ACS and age-matched 16 healthy male (control) were included in the study. The diagnosis of ACS was based on the clinical presentation, ECG and serum cardiac biomarkers. These patients were grouped as acute myocardial infarction with ST-segment elevation subtype (STEMI, $n=24)$ and non-ST-segment elevation myocardial infarction subtype (NSTEMI, $n=9$ ). Serum and salivary antioxidant activity (AOA) were determined spectrophotometrically. Results: Antioxidant defense, as reflected by lower total AOA in saliva and serum, was found to be significantly attenuated in patients with ACS as compared to control subjects, indicating an increased oxidative stress. The decrease in AOA was more conspicuous in patients with STEMI as compared to non-NSTEMI suggesting more oxidative stress in the former is associated with the severity of the disease, including tissue necrosis in STEMI patients. Drug treatment to ACS patients for a short-term period (4-6 days) elicited no significant improvement in their AOA. However, ACS patients receiving longterm drug treatment (4-5 weeks) exhibited a significant increase in AOA as well as an improvement in the clinical status. Conclusion: This preliminary study supports the role of oxidative stress in the pathogenesis of ACS and the use of salivary and serum AOA as a potential marker of the redox status and disease severity.
\end{abstract}

KEYWORDS: Antioxidants, saliva, serum, acute coronary syndrome

\section{INTRODUCTION}

Acute coronary syndrome (ACS) is one of the most common and life-threatening illnesses all over the world. ACS covers the clinical spectrum of acute myocardial ischemia, including unstable angina, acute myocardial infarction without ST-segment elevation and ST-segment elevation myocardial infarction. ${ }^{1}$ It is characterized by a major hemodynamic insult to a person's homeostatic balance in the normal physiological system. It is well established that atherosclerotic disease of the epicardial coronary arteries is the most common cause of ACS.

Oxidative stress has been implicated in the pathogenesis of many diseases, including cardiovascular diseases. ${ }^{2,3}$ An imbalance between

Corresponding author:

Basheer Ali, Ph.D.

Department of Pharmacology and Therapeutics

C.S.M. Medical University

Lucknow 226003 (U.P.), India

Phone: +919415026156

Email: basheer.kgmu@gmail.com cellular production of free radicals and the ability of cells to defend against them is referred to as oxidative stress. ${ }^{4}$ Cells and body fluids have an array of protective antioxidants both for preventing the production of free radicals and for scavenging them. ${ }^{5}$ Much less is understood about the redox status of the patients with ACS. The present study was, therefore, designed to evaluate the redox status of patients suffering from ACS prior to and following therapy as compared to age-matched healthy individuals by monitoring the antioxidant potential of serum and saliva as biomarker of oxidative stress and evaluating their possible correlation with the clinical status of the patients.

\section{MATERIAL AND METHODS \\ Subjects}

A total of 33 patients (29-78 year old, male) with ACS undergoing treatment in the Department of Cardiology and age-matched 16 healthy male (Control) were included in the study. The diagnosis of ACS was based on the clinical presentation, ECG and serum cardiac biomarkers. These patients were grouped as acute myocardial infarction with ST-segment elevation subtype (STEMI, $\mathrm{n}=24$ ) and non-ST-segment elevation myocardial infarction subtype (NSTEMI, $n=9$ ). A complete clinical work up of the patients was done and 
case history was recorded. Patients with stable angina pectoris, suffering from multiple medical illness or those suffering from mental illness were excluded from the study. Due to patient's noncompliance with regular treatment and/or hospital attendance, ten patients receiving the treatment were followed up and were divided into short-term follow-up (4-6 days) and long - term follow up (4-5 weeks) groups on the basis of the duration of therapy. Since therapy varied from patient to patient the post-treatment group was divided on the basis of the duration of therapy and not on the type of drug therapy. The medication in general comprised of streptokinase, aspirin, antithrombotic drugs, beta blockers, angiotensin-converting enzyme inhibitors, lipid lowering drugs, etc. depending on the patient's requirement. Written informed consent to participate in the study was taken from the patients and controls.

\section{Sample Collection}

Venous blood (1.5-2 $\mathrm{ml}$ ) was collected in plain vials, serum was harvested and stored at $4-80 \mathrm{C}$. The patients and controls were asked to rinse the mouth with water, and no oral intake was allowed at least 30 minutes prior to saliva collection. The saliva $(1-1.5 \mathrm{ml})$ was collected in multiple spit within 10 minutes, centrifuged at $2,000 \mathrm{~g} \times 10$ minutes and the clear saliva was stored at $4-80 \mathrm{C}$. The antioxidant activity in serum and saliva was determined on the same day of sample collection.

Determination of Antioxidant activity (AOA) AOA in saliva/serum was determined according to the method of Koracevic et al.6 The method was based on the principle that Fe-EDTA complex reaction with hydrogen peroxide (Fenton reaction) generates superoxide and hydroxyl radicals. These reactive oxygen species degrade benzoate resulting in the release of thio-barbituric acid reactive substances (TBARS). The inhibitory potential of saliva/serum against TBARS production is then determined as antioxidant activity (represented as mmole/l) using standard antioxidant, uric acid, as describe earlier. ${ }^{7}$ The reac $\neg$ tion mixture containing $0.5 \mathrm{ml}$ of phosphate buffer $(100 \mathrm{mmole} / \mathrm{l}, \mathrm{pH} 7.4), 0.5 \mathrm{ml}$ of sodium benzoate ( $10 \mathrm{mmole} / \mathrm{l}), 0.2 \mathrm{ml}$ of Fe-EDTA ( $2 \mathrm{mmole} / \mathrm{l}$ EDTA + 2 mmole/l of Fe[NH4]2SO4, 0.2 ml of H2O2 (10 mmole/l) and $0.01 \mathrm{ml}$ of saliva/serum was incubated for 60 minutes at $37^{\circ} \mathrm{C}$. The reaction was stopped by addition of $1 \mathrm{ml}$ of $20 \%$ acetic acid. One $\mathrm{ml}$ of thiobarbituric acid (TBA) solution $(0.8 \%$ in 50 mmole/l $\mathrm{NaOH}$ ) was added, and the solution was heated for 10 minutes at $100^{\circ} \mathrm{C}$. The absorbance of the pink color thus formed was estimated spectrophotometrically at $532 \mathrm{~nm} .0 .01 \mathrm{ml}$ of uric acid (1mmole// in $5 \mathrm{mmole} / \mathrm{l}$ $\mathrm{NaOH}$ ) was used as the standard antioxidant for determining the AOA of unknown samples. Proper blanks were run under similar experimental conditions. The AOA of each sample was calculated as follows:

$$
\text { AOA (mmole } / \mathrm{l})=\frac{(\mathrm{CU})(\mathrm{K}-\mathrm{A})}{(\mathrm{K}-\mathrm{UA})}
$$

Where $\mathrm{K}=$ absorbance of control; $\mathrm{A}=$ absorbance of sample; $U A=$ absorbance of uric acid solution; $C U=$ concentration of uric acid (mmole/l).

The serum and salivary AOA of the patients were expressed as the mean + S.E. Statistical significance of the difference between the groups was compared by Analysis of Variance (ANOVA) and p value less than 0.05 was considered statistically significant. ${ }^{7}$

\section{RESULTS}

The total AOA in saliva of ACS patients (STEMI + NSTEMI) was significantly lower $(p<0.01)$ than that observed in the control group (Table 1). The salivary AOA in the patients with ACS subtypes, STEMI, and NSTEMI was significantly lower than the control AOA ( $p<0.01$ and $<0.05$, respectively). Similarly, the levels of serum AOA in combined ACS patients $(p<0.01)$ as well as in both STEMI $(p<0.01)$ and NSTEMI $(p<$ 0.05 ) groups of patients separately were significantly lower than that found in the control group. The decrease in the mean $A O A$ in patients as compared to controls was slightly higher in the saliva than in serum (29\% versus $22 \%$ ). Out of 33 ACS patients, only eight presented with some form of co-morbid illness like diabetes mellitus, hypertension and heart failure but the AOA in this small number of patients was not much different as compared to those without any co-morbid illness and, therefore, all included in the study.

The AOA in the saliva of patients after 4-6 days of drug treatment was not significantly different from the pretreatment levels of AOA (Table 2). However, after long-term drug treatment (4-5 weeks), the level of AOA increased significantly as compared to pretreatment level but was still significantly lower

Table 1. Antioxidant activity of saliva and serum in control and ACS patients

\begin{tabular}{|c|c|c|}
\hline \multirow{2}{*}{ Group } & \multicolumn{2}{|c|}{ AOA (mmole/l) } \\
\hline & Saliva & Serum \\
\hline $\begin{array}{l}\text { Control } \\
\text { ACS patients }\end{array}$ & $\begin{array}{l}1.35 \pm 0.05 \quad(n=16) \\
0.96 \pm 0.06^{* *}(n=33)\end{array}$ & $\begin{array}{l}1.56 \pm 0.08(n=13) \\
1.22 \pm 0.06^{* *}(n=31)\end{array}$ \\
\hline $\begin{array}{l}\text { Subtypes } \\
\text { STEMI } \\
\text { NSTEMI }\end{array}$ & $\begin{array}{l}0.93 \pm 0.06^{* *}(n=24) \\
1.07 \pm 0.14^{*}(n=9)\end{array}$ & $\begin{array}{l}1.17 \pm 0.09^{* *}(n=22) \\
1.24 \pm 0.11^{*}(n=9)\end{array}$ \\
\hline
\end{tabular}

Values are the mean \pm SE. $n=$ number of subjects; ${ }^{*} p<0.05$ as compared to control; ${ }^{* *} p<0.01$ as compared to control 
Table 2. Effect of shot-term and long-term drug treatment on AOA of saliva and serum in ACS patients

\begin{tabular}{|c|c|c|c|c|c|}
\hline \multirow[t]{2}{*}{ Group } & \multirow[b]{2}{*}{ Control } & \multicolumn{2}{|c|}{ Short-term treatment } & \multicolumn{2}{|c|}{ Long-term treatment } \\
\hline & & Pretreatment & Post-treatment & Pretreatment & Post-treatment \\
\hline Saliva & $\begin{array}{l}1.35 \pm 0.05 \\
(n=16)\end{array}$ & $\begin{array}{l}0.88 \pm 0.09^{*} \\
(n=4)\end{array}$ & $\begin{array}{c}0.92 \pm 0.09^{*} \dagger \\
(n=4)\end{array}$ & $\begin{array}{l}0.86 \pm 0.06^{*} \\
(n=6)\end{array}$ & $\begin{array}{c}1.08 \pm 0.07^{*} \# \\
(n=6)\end{array}$ \\
\hline Serum & $\begin{array}{c}1.56 \pm 0.08 \\
(n=13)\end{array}$ & $\begin{array}{c}1.09 \pm 0.07^{*} \\
(n=4)\end{array}$ & $\begin{array}{c}1.15 \pm 0.08^{*} \dagger \\
(n=4)\end{array}$ & $\begin{array}{c}1.14 \pm 0.05^{*} \\
(n=6)\end{array}$ & $\begin{array}{c}1.40 \pm 0.08 \# \# \\
(n=6)\end{array}$ \\
\hline
\end{tabular}

\author{
Values are the mean \pm S.E. $n=$ number of subjects \\ * $\quad \mathrm{p}<0.01$ as compared to control \\ \# $\quad \mathrm{p}<0.05$ as compared to pretreatment \\ \# \# $\mathrm{p}<0.01$ as compared to pretreatment \\ $\dagger \quad$ Statistically insignificant as compared to pretreatment group of patients
}

than that of control subjects. Likewise, serum AOA did not change after short-term drug treatment, but increased significantly after long-term treatment and restored almost to control values.

\section{DISCUSSION}

Evidence from experimental and clinical studies has suggested the role of oxidative stress in the pathogenesis of cardiovascular disease $e^{2,3}$ and heart dysfunction. ${ }^{8}$ Total AOA represents the sum of activities of antioxidants present in the tissue/body fluid. ${ }^{6,9}$ The composition of saliva can be compared to that of blood over a wide variety of analytes, and saliva is also armed with various defense mechanisms, including antioxidant system. ${ }^{10,11}$ Ex-vivo experiments have demonstrated that excess free radical generation or impaired antioxidant function adversely affects myocardial function. ${ }^{12-14}$ Increased oxidative stress and depressed antioxidant system in patients with acute myocardial infarction has been reported recently. ${ }^{15}$ There is evidence that oxidative stress plays an important role in congestive heart failure and ventricular arrhythmias. ${ }^{16}$ However, much less is known about the possible role of oxidative stress in the pathogenesis of ACS.

The present study shows a significantly decreased salivary AOA in patients with ACS as compared to that in healthy controls. In line with this, the AOA of serum in patients was also significantly lower as compared to control value. These observations suggest an increased oxidative stress in patients with ACS. A greater decrease in salivary AOA may be due to lesser antioxidant buffering capacity of saliva. It may attribute to smaller volume of saliva secreted as compared to that of serum, which represents the systemic circulation directly in contact with the necrotic tissue and has a greater amount of available antioxidants. A greater decrease in both salivary and serum AOA of patients with STEMI compared to those with NSTEMI could be due to the fact that STEMI is much more severe disease condition associated with probably greater oxidative cell damage than NSTEMI.
The pattern of attenuation of serum AOA was similar to that found in saliva of patients with ACS indicating that salivary AOA could be used as a biomarker of oxidative stress and an alternative to serum AOA. However, it is difficult to reach any definite conclusion on the utility of serum versus salivary AOA as a biomarker based on a small number of patients in the present study; but, it is considerably easier, safer, non-invasive and more economical to collect saliva samples as compared to the blood specimen. Hence, salivary AOA deserves further validation with more patients. To the best of our knowledge, the current study is a pioneering effort to assess oxidative stress in patients with ACS using salivary AOA as a biomarker.

It is pertinent to mention here that there was a variation in the drug treatment schedule; therefore, the post-treatment group was divided based on the duration of therapy and not on the basis of the drugs employed in the treatment into short-term and long-term follow-up groups. The short-term follow-up group of patients showed variable or insignificant clinical improvement, whereas the long-term drug therapy showed significant clinical improvement in terms of cardiac function markers as well as improvement in the clinical symptoms like chest pain, dyspnea, diaphoresis, nausea and anxiety. Remarkably, this correlated well with the status of $A O A$ in that short-term drug treatment to patients did not show any restoration of either salivary or serum AOA, but long-term treatment caused an improvement in AOA. It is likely that the pathophysiological changes and associated decrease in AOA occurring during the course of the disease requires more recovery period of time to restore to control values.

Prospective epidemiological studies have suggested that high doses of antioxidant vitamins C, A and E could prevent or delay cardiovascular disease. ${ }^{17}$ The percentage and severity of EMI and triple vessel disease goes down with increasing serum levels of endogenous antioxidants.18 However, the results from clinical trials of antioxidant supplementation 
in cardiovascular disease have been inconclusive. ${ }^{19-21}$ At present, it is difficult to conclude whether the attenuation of antioxidant capacity seen in our patients with ACS has a role in the etiology of the disease, or it is the consequence of the disease. In either case, the proposed supplementation of antioxidants may improve the redox status including cardiovascular functions and possibly contain any further oxidative tissue damage. The present study is likely to offer a new dimension for future research towards elucidation of the role of oxidative stress in the pathogenesis of ACS and evaluation of supportive therapeutic use of antioxidants.

\section{CONCLUSION}

This preliminary study shows a significantly decreased serum and salivary AOA in patients with ACS as compared to that in healthy controls. A greater decrease in both salivary and serum AOA of patients with STEMI compared to those with NSTEMI could be due to the fact that STEMI is a more severe disease condition associated with greater oxidative cell damage than NSTEMI. Remarkably, short-term (4-6 days) drug treatment to patients did not show any restoration of either salivary or serum AOA, but longterm treatment (4-5 weeks) caused an improvement in both $\mathrm{AOA}$ and clinical status. These findings support the role of oxidative stress in the pathogenesis of ACS and the use of salivary and serum AOA as potential markers of the redox status and disease severity.

Conflicts of interest: none

\section{REFERENCES}

1. Zimetbaum PJ, Josephson ME. Use of the electrocardiogram in acute myocardial infarction. N Engl J Med 2003;348:933-40.

2. Dhalla NS, Temsah RM, Netticadan T. Role of oxidative stress in cardiovascular disease. J Hypertension 2000;18:655-73.

3. Griendling KK, FitzGerald GA. Oxidative stress and cardiovascular injury: Part II : animal and human studies. Circulation 2003;108:2034-40.

4. Sies H. Oxidative stress: From basic research to clinical application. Am J Med 1991;91:31S-38S.

5. Yu BP. Cellular defences against damage from reactive oxygen species. Physiol Rev 1994; 74:139-62.

6. Koracevic D, Koracevic G, Djordjevic V, Andrejevic S, Cosic V. Methods for the measurement of antioxidant activity in human fluids. J Clin Pathol 2001;54:356-61.

7. Ruchi K, Anil Kumar S, Sunil G, Bashir A, Prabhat S. Antioxidant activity in children with ADHD - a comparison in untreated and treated subjects with normal children. International Medical Journal Malaysia 2011;10:31-5.

8. Singal PK, Khaper N, Palace V, Kumar D.
The role of oxidative stress in the genesis of heart disease, Cardiovasc Res 1998;40:42632.

9. Ziobro A. Bartosz G. A comparison of the total antioxidant capacity of some human body fluids. Cell Mol Biol 2003;8:415-9.

10. Moore S, Calder KA, Miller NJ, Rice-Evans CA. Antioxidant activity of saliva and periodontal disease. Free Radic Res 1994;21:417-25.

11. Battino M, Ferreiro MS, Gallardo I, Newman $\mathrm{HN}$, Bullon P. The antioxidant capacity of saliva: Review. J Clin Peridontol 2002;29:189-94.

12. Goldhaber JI, Ji S, Lamp ST, Weiss JN. Effects of exogenous free radicals on electromechanical function and metabolism in isolated rabbit and guinea pig ventricle. Implications for ischemia and reperfusion injury. J Clin Invest 1989;83:1800-9.

13. Blaustein AS, Schine L, Brooks WW, Fanburg BL, Bing $\mathrm{OH}$. Influence of exogenously generated oxidant species on myocardial function. Am J Physiol 1986;250:595-9.

14. Burton KP, McCord JM, Ghai G. Myocardial alterations due to free radical generation, Am J Physiol 1984;246:776-83.

15. Patil N, Chavan V, Karnik ND. Antioxidant Status in patients with acute myocardial infarction. Ind J Clin Biochem 2007; 22:45-51.

16. Mak S, Newton GE. The oxidative stress hypothesis of congestive heart failure. Chest 2001; 120:2035-46.

17. Knekt P, Ritz J, Pereira MA, et al. Antioxidant vitamins and coronary heart disease risk: a pooled analysis of 9 cohorts. Am J Clin Nutr 2004; 80:1508-20.

18. Verma VK, Ramesh V, Tewari S, et al. Role of bilirubin, vitamin $C$ and cerruloplasmin as antioxidants in coronary artery disease, Ind $\mathrm{J}$ Clin Biochem 2005;20:68-74.

19. Asplund K. Antioxidant vitamins in the prevention of cardiovascular disease: a sys temic review. J Intern Med 2002;251:372-92.

20. Pham DQ, Plakogiannis R. Vitamin E supplementation in cardiovascular disease and cancer prevention: Part 1. Ann Pharmacother 2005;39:1870-78.

21. Traba MG. Heart disease and single vitamin supplementation. Am J Clin Nutr 2007;85:293-9. 